\title{
Germinal Matrix Hemorrhage of the Newborn
}

National Cancer Institute

\section{Source}

National Cancer Institute. Germinal Matrix Hemorrhage of the Newborn. NCI Thesaurus.

Code C99138.

Bleeding into the thick layer of immature cells under the ependymal lining at the ventrolateral aspect of the lateral cerebral ventricles of a newborn. 